\title{
Percepção da mulher sobre as orientações do enfermeiro no exame de prevenção de câncer de colo uterino
}

Woman's perception of nursing guidelines on examination of uterine cervical cancer prevention Percepción de la mujer de las directices de enfermería en el examen de la prevención del cáncer de cuello uterino

Gleyson Leandro Leão Santos ${ }^{1}$; Jomar Maia Xavier² ${ }^{2}$ Patrick Leonardo Nogueira da Silva ${ }^{3}$; Rogério Gonçalves da Rocha ${ }^{4}$; Tadeu Nunes Ferreira ${ }^{5}$

\section{Resumo}

O câncer do colo uterino representa uma grave problemática na saúde pública que atinge parte das mulheres em todo o mundo. Este estudo objetivou identificar a percepção da mulher sobre as orientações do enfermeiro no exame de prevenção cérvico uterino. Trata-se de um estudo descritivo, transversal, com abordagem qualitativa. A amostra foi composta por 10 mulheres residentes em uma área de abrangência de uma Estratégia Saúde da Família de Minas Gerais. A coleta de dados foi realizada por meio de uma entrevista semiestruturada. Os depoimentos foram gravados, transcritos na íntegra e analisados por meio da Análise de Conteúdo. Os resultados mostram que algumas mulheres tinham conhecimentos prévios sobre o câncer cérvico uterino. Estes eram mais bem difundidos e entendidos na educação em saúde por meio de recursos audiovisuais. Mulheres que realizaram o exame preventivo se sentiram mais femininas e apresentaram sua autoestima elevada. Identificou-se que muitas mulheres dão importância ao exame preventivo devido às campanhas realizadas pela Unidade de Saúde. Portanto, faz-se necessária a sensibilização das mulheres quanto ao exame de prevenção como forma de proteção individual de modo a evitar a exposição das mulheres aos elementos nocivos e gerar qualidade de vida.

Descritores: Percepção, Câncer, Orientações.

\section{Abstract}

The cervical cancer is a serious problem in public health that affects the part of women worldwide. This study aimed to identify the perception of women on the nurse's guidelines on examination of uterine cervical prevention. This is a descriptive, cross-sectional study with a qualitative approach. The sample consisted of 10 women living in a catchment area of a Health Strategy Minas Gerais family. Data collection was performed using a semi-structured interview. The interviews were recorded, transcribed verbatim and analyzed using content analysis. Results show that women had some prior knowledge of uterine cervical cancer. These were better disseminated and understood in

\footnotetext{
${ }^{1}$ Graduação em Enfermagem pelas Faculdades Unidas do Norte de Minas (FUNORTE). Avenida Osmane Barbosa, $\mathrm{n}^{\circ}$ 11.111, JK, Montes Claros, MG, Brasil. CEP: 39.404-549. E-mail: gleysonleao@ hotmail.com

${ }^{2}$ Graduação em Enfermagem pelas Faculdades Unidas do Norte de Minas (FUNORTE). Avenida Osmane Barbosa, $n^{\circ}$ 11.111, JK, Montes Claros, MG, Brasil. CEP: 39.404-549. E-mail: tadeu-nunes@ hotmail.com

${ }^{3}$ Especialista em Saúde da Família e Didática e Metodologia do Ensino Superior pela Universidade Estadual de Montes Claros (UNIMONTES). Enfermeiro da Secretaria Municipal de Saúde de Espinosa (MG). Avenida Juscelino Kubitscheck, s/nº, Santa Tereza, Espinosa, MG, Brasil. CEP: 39.510-000. E-mail: patrick_mocesp70@ hotmail.com

${ }^{4}$ Graduação em Enfermagem pela Universidade Estadual de Montes Claros (UNIMONTES). Enfermeiro da Secretaria Municipal de Saúde de Montes Claros (MG). Avenida Dulce Sarmento, no 2076, Monte Carmelo, Montes Claros, MG, Brasil. CEP: 39.401-485. E-mail: rogeriorocha81@yahoo.com.br

${ }^{5}$ Mestrando em Tecnologia da Informação (Área de Concentração em Biologia Molecular Estrutural e Linha de Pesquisa em Biologia Computacional) pela Única Educacional (ÚNICA). Professor das Faculdades Unidas do Norte de Minas (FUNORTE). Avenida Osmane Barbosa, nº 11.111, JK, Montes Claros, MG, Brasil. CEP: 39.404-549. E-mail: tadeu-nunes@hotmail.com
} 
ISSN 2179-6750

health education through audio-visual aids. Women who underwent the screening test felt more feminine and presented their high self-esteem. It was identified that many women give importance to preventive screening due to campaigns by the health unit. Therefore, it is necessary to raise awareness of women and to take prevention as a means of individual protection in order to avoid exposure of women to the elements harmful and generate quality of life.

Key-words: Perception, Cancer, Guidelines.

\section{Resumen}

El cáncer de cuello uterino es un problema serio en la salud pública que afecta a la parte de las mujeres en todo el mundo. Este estudio tuvo como objetivo identificar la percepción de las mujeres sobre las directrices de la enfermera en el examen de la prevención de cérvix uterino. Se trata de un estudio descriptivo, transversal, con un enfoque cualitativo. La muestra consistió en 10 mujeres que viven en un área de influencia de una familia Estrategia de Salud de Minas Gerais. La recolección de datos se realizó mediante una entrevista semiestructurada. Las entrevistas fueron grabadas, transcritas textualmente y se analizaron mediante análisis de contenido. Los resultados muestran que las mujeres tenían algún conocimiento previo de cáncer de cuello uterino. Estos fueron mejor difusión y comprensión en la educación sanitaria a través de medios audiovisuales. Las mujeres que se sometieron a la prueba de detección se sentían más femenino y presentaron su alta autoestima. Se identificó que muchas mujeres dan importancia a la detección preventiva debido a las campañas por la unidad de salud. Por lo tanto, es necesario aumentar la conciencia de la mujer y aplicar la prevención como un medio de protección con el fin de evitar la exposición de las mujeres a los elementos perjudiciales y generar calidad de vida.

Palabras-claves: Percepción, Cáncer, Directrices.

\section{Introdução}

O câncer do colo uterino (CCU) representa uma grave problemática na saúde pública que atinge parte das mulheres em todo o mundo. A sua incidência é duas vezes mais elevada em países com grau de desenvolvimento menor em comparação aos países mais desenvolvidos, e o Brasil responde a uma taxa expressiva desses números. A distribuição de casos novos, em consonância com a localização primária, é muito diferenciada entre os estados e capitais brasileiros. Não se considerando os tumores de pele não melanoma, o câncer cervical é a de maior incidência na região Norte (23/100.000), nas regiões Centro-Oeste (20/100.000) e Nordeste (18/100.000), e o segundo lugar mais frequente é nas regiões Sul (21/100.000) e Sudeste (16/100.000), a terceira posição ${ }^{1}$.

O CCU configura-se atualmente como um sério problema de saúde pública, visto que no ano de 2012 e 2013, aproximadamente 18 mil novos casos foram encontrados, sendo a neoplasia citada acima umas das mais frequentes na população feminina, superada apenas pelo câncer de pele (não melanoma) e o câncer de mama ${ }^{1}$. Estudos do Instituto Nacional do Câncer (INCA) referentes à prevalência do câncer nas mulheres indicam que o CCU é uma das modalidades mais incidentes e o segundo em mortalidade nestas mulheres. Tais taxas, além de despertar a iniciativa do presente 
ISSN 2179-6750

estudo, também possibilitam uma observação da gravidade e a complexidade da problemática do câncer ${ }^{2}$.

Ressalta-se ainda que, dentre os tipos de câncer citados, o de útero é o que apresenta um dos mais elevados potenciais para prevenção e cura podendo atingir até $100 \%$, quando diagnosticado precocemente, e ainda pode ser tratado em nível ambulatorial em cerca de $80 \%$ dos casos ${ }^{3}$. Quanto à evolução dos estudos voltados para os riscos vinculados ao CCU, verificou-se que as mulheres com baixas condições socioeconômicas, início precoce da atividade sexual, multiplicidade de parceiros sexuais e com o hábito tabagista, estão entre o número das que já tiveram alguma infecção pelo Papiloma Vírus Humano (HPV), de forma a entender que as informações de prevenção primária e secundária têm ligação direta com a prevenção contra o $\mathrm{CCU}^{4}$.

O histórico de doenças sexualmente transmissíveis (DST), sobretudo na exposição ao HPV, é um fator de risco de ampla significância para o desenvolvimento do CCU. Estando o HPV envolvido em $99 \%$ dos casos de CCU, estudos vêm evidenciando um papel relevante na transformação das células cervicais e o desenvolvimento de neoplasia. Sabe-se que o CCU é um câncer que apresenta índice de cura, se detectado precocemente, e dependendo das condições de vida e saúde dessas mulheres ${ }^{5}$.

O CCU pode ser prevenido, se for detectado precocemente; isso quer dizer que é uma prevenção secundária, uma vez que estaria sendo interceptada, através de métodos diagnósticos, a evolução de possíveis lesões malignas ${ }^{6}$. Um exemplo de prevenção é o exame citopatológico (Papanicolau). Ele é o exame de rastreamento que deve ser efetuado nas mulheres com idade entre 25 a 64 anos e que já possuam vida sexual ativa. Esta faixa etária foi determinada em consonância com os principais programas de nível internacional, tendo em vista que, possui prioridade devido ao maior número de ocorrências das lesões de alto grau que podem ser tratadas efetivamente para não progredirem para a neoplasia ${ }^{1}$. A periodicidade de que este exame deve ser realizado é a cada três anos, após dois exames normais respectivos realizados com um intervalo de um ano.

$\mathrm{Na}$ ótica de muitos profissionais de saúde, o exame representa um procedimento simples, rápido, rotineiro e indolor. Por outro lado, na óptica das mulheres, ele pode ser enxergado como um procedimento psicologicamente e fisicamente agressivo, pois como a mulher que procura o serviço de saúde traz consigo suas bagagens sociais, culturais, familiares e religiosas o que faz com que haja interpretações diversas sobre o mesmo exame. Acredita-se que o profissional de enfermagem deve manter uma postura habitual de sensibilização para com as mulheres, no intuito de que elas continuem buscando realizar o exame de Papanicolau, além disso, uma busca consciente ${ }^{5}$.

É relevante salientar que as práticas de Educação em Saúde necessitam ser reforçadas, por 168 
ISSN 2179-6750

meio da participação de toda Estratégia de Saúde da Família (ESF), já que muitas das razões para a não realização do Papanicolau podem ser resolvidas de uma forma fácil por meio dessa prática. As ESF, tidas como porta de entrada nos serviço de saúde, apresentam potencialidades para qualificar a prática da Prevenção de Câncer de Colo do Útero (PCCU) e promover uma maior integralidade das ações em saúde, utilizando estratégias para atrair a população ${ }^{6}$.

A Educação em Saúde é uma estratégia metodológica que deve ser usada como uma ferramenta de intervenção, visto que fornece conhecimento sobre o tema estabelecido e oferece interação entre as participantes, por meio de ações educativas de prevenção, proteção e recuperação da saúde, com objetivo de ampliar a qualidade de vida (QV). Suas ações são realizadas em campanhas, palestras, projetos e oficinas, com finalidade de reforçar práticas educativas para a prevenção de doenças e aquisição de posturas saudáveis. Entretanto, para que as mulheres participem das reuniões é importante ressaltar a interação desses dois atores (usuário e profissional de saúde), o contato de ambos quando não alcança tal interação com o usuário, dificilmente verificará o retorno da paciente em procurar o serviço, diminuindo assim a adesão das mulheres nas reuniões de Educação em Saúde ${ }^{7}$. Logo, o diálogo é essencial em todas as ocasiões do encontro com a mulher, tanto no serviço de saúde da família, quanto fora dele ${ }^{6}$, pois não acredita que se chegará a uma sensibilização se não por meio de dialogo.

É essencial que os serviços de saúde norteiem a importância do PCCU, já que a realização periódica pode encurtar a mortalidade por CCU na população de risco, além de empregar as atividades de Educação em Saúde conseguirá estabelecer um vínculo entre cliente e profissional, gerando confiança e respeito do paciente como um ser holístico ${ }^{6}$.

No cenário da prevenção do CCU, a atuação do enfermeiro nas ESF se revelou de importância fundamental, e as suas atividades são desenvolvidas em múltiplas dimensões, entre elas: realização das consultas de enfermagem e do exame de Papanicolau, ações educativas diversas junto à equipe de saúde e comunidade, gerenciamento e contatos para o provimento de recursos materiais e técnicos, controle da qualidade dos exames, verificação, comunicação dos resultados e encaminhamentos para os devidos procedimentos quando necessário. Diante desta realidade, passase a analisar os problemas da percepção da mulher sobre o exame de Papanicolau, e o papel da equipe de saúde, juntamente com o enfermeiro, é fundamental para transmitir e orientar a paciente sobre a importância da prevenção ${ }^{8,5}$.

O problema posto para investigação tem a seguinte questão norteadora: Qual a percepção da mulher sobre as orientações do enfermeiro no exame de PCCU? Portanto, o presente estudo objetivou identificar a percepção da mulher sobre as orientações do enfermeiro no exame de PCCU. 


\section{Método}

Trata-se de um estudo transversal, com abordagem qualitativa, realizada na Estratégia Saúde da Família Vila Anália localizada no município de Montes Claros, norte de Minas Gerais. A amostra foi composta por 10 mulheres residentes na área de abrangência. A pesquisa qualitativa ocupa-se do universo dos significados trabalhando com um espaço mais profundo das relações que não podem ser reduzidos a observação de variáveis ${ }^{9}$.

A amostra foi definida por saturação na qual adotou-se os seguintes critérios de inclusão: indivíduos maiores de 18 anos com vida sexual ativa para a realização do PCCU; indivíduos que possuíam habilidade para se expressar facilmente por palavras; indivíduos que possuíam habilidade para perceber e expressar seus sentimentos e emoções sem vergonha ou inibição; indivíduos que tiveram experiência recente com a realização do exame de Papanicolau.

Utilizou-se como instrumento de coleta de dados a técnica de entrevista semiestruturada. Os depoimentos foram registrados por meio de um gravador no qual os mesmos foram transcritos na íntegra e posteriormente analisados e discutidos. As participantes foram identificadas no estudo com nomes de pedras preciosas a fim de preservar a sua identidade de forma a respeitar o sigilo e o anonimato da pesquisa.

Os preceitos éticos foram respeitados e seguidos conforme a Resolução 466 de 12 de dezembro de 2012, do Conselho Nacional de Saúde (CNS) na qual regulamenta a pesquisa envolvendo seres humanos. O estudo foi apreciado e aprovado pelo Comitê de Ética em Pesquisa das Faculdades Unidas do Norte de Minas (CEP FUNORTE) sob parecer n ${ }^{\circ}$ 804.968/2014, Certificado de Apresentação para Apreciação Ética (CAAE): 34944614.9.0000.5141.

O tratamento dos dados se deu pela análise de conteúdo ${ }^{10}$, sendo esta um método que se baseia em um conjunto de técnicas de análise das comunicações com procedimentos sistemáticos para a descrição do conteúdo das mensagens ${ }^{11}$. Para o tratamento baseou-se ainda na estrutura que utiliza da análise temática do material, sendo organizado e estruturado seguindo as fases: préanálise, exploração do material e tratamento dos resultados ${ }^{9}$. Os resultados foram descritos por categorizações, expondo os achados encontrados.

Após leituras flutuantes das entrevistas, foram realizadas as identificações dos códigos e categorização dos depoimentos que resultaram nas seguintes categorias: "O aprendizado sobre câncer de colo de útero deve ser visual", "Fazer o exame revela a feminilidade", e "as orientações e o exame são focados na prevenção", conforme Figura 1. 


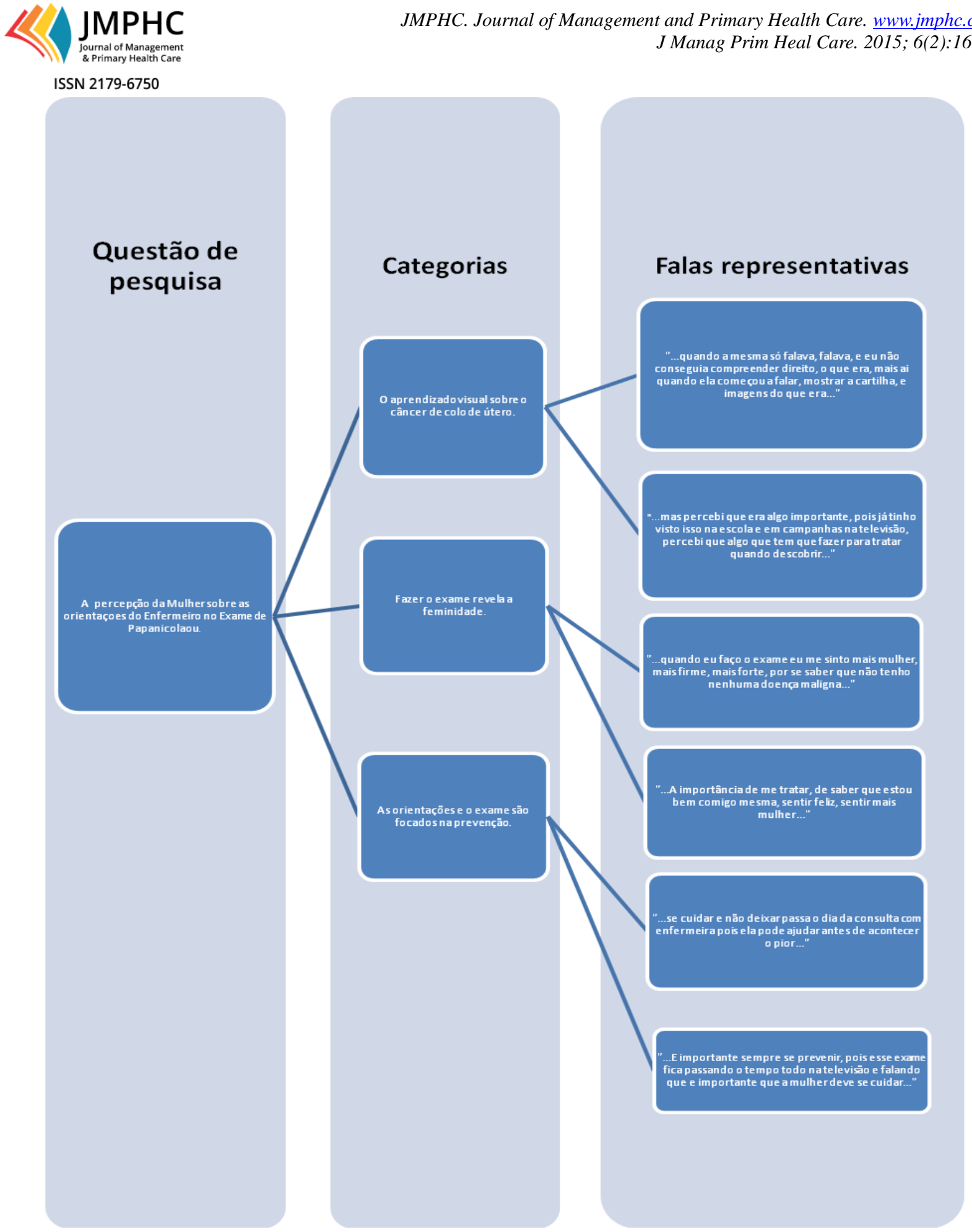

Figura 1. Representação gráfica da problemática do estudo conforme as categorias e depoimentos representativas. Fonte: Elaboração Própria, 2014.

\section{Resultados e Discussão}

$\mathrm{O}$ aprendizado sobre $\mathrm{CCU}$ deve ser visual

Nesta categoria observou-se que algumas mulheres dependem de estratégias de ensino que se baseiam em imagens, pois o aprendizado das mesmas conforme observado nos depoimentos é visual. 
ISSN 2179-6750

As estratégias de ensino devem ser utilizadas também como base para as orientações em saúde, e isto deve ser parte do processo de trabalho do enfermeiro, no que se refere ao momento do contato com a mulher no exame de PCCU. Estas estratégias configuram-se como elementos fundamentais já que permitem uma assimilação melhor das informações sobre a doença, seus riscos e formas de prevenção.

Através da história da humanidade observa-se que a imagem sempre foi utilizada como forma de aprimorar o aprendizado e de fixação do conteúdo oral; é o que pode ser visto, por exemplo, na transmissão da história e nos relatos de tempos anteriores à escrita. No caso das ciências médicas nota-se atualmente um encontro com a ciência da imagem sendo até possível falar de um positivismo da imagem com a utilização de vídeos, fotos e outros recursos com a intenção de tornar o ensino em saúde mais objetivo através do discurso "ilustrado" do profissional de saúde ${ }^{12}$.

Através das entrevistas percebeu-se que o discurso das mulheres reforça a ideia de que a educação em saúde deve ser trabalhada com o uso de recursos audiovisuais. Notou-se também que algumas mulheres tinham conhecimentos prévios adquiridos pela observação de campanhas televisivas ou outras.

[...] quando a mesma só falava, falava e eu não conseguia compreender direito, o que era mais aí quando ela começou a falar e mostrar a cartilha, e imagens do que era, e qual a importância, eu percebi que era uma coisa de que havia de tratar preventivamente e que se não tratasse, era possível já de se descobrir uma doença em que não havia mais cura, não só DST's, mais também o câncer de colo. (Ametista)

O depoimento acima da paciente Ametista mostra que enquanto o enfermeiro abordava de forma teórica e discursiva o assunto não havia compreensão favorável, mas com o uso de imagens nota-se uma ampliação de sua percepção que é na verdade o que se espera do processo de educação em saúde. À medida que o indivíduo conhece mais e assimila melhor as informações consegue compreender a real importância das estratégias preventivas e é neste ponto que a educação em saúde posiciona-se já que através dela deve-se buscar a construção de um conhecimento que leve a mudanças de comportamento para a saúde dos indivíduos. Isto só ocorre quando se conhece a real necessidade da população e se trabalha de forma específica com as particularidades da clientela atendida, é possível perceber estas particularidades no depoimento seguinte:

[...] mas percebi que era algo importante, pois já tinha visto isso na escola e em campanhas na televisão percebi que é algo que tem que fazer para tratar quando descobrir 
O depoimento da paciente Rubi mostra que já havia um conhecimento prévio sobre o assunto adquirido na escola e em campanhas veiculadas na televisão, nota-se que o processo de educação em saúde neste caso deve considerar o que a cliente já possuía de informação e trabalhar este conteúdo afirmando-o ou quem sabe até desconstruindo conceitos errôneos que poderiam até mesmo prejudicá-la.

Para implantar a Educação em Saúde no processo de saúde/doença e para construir uma prática educativa resolutiva, é indispensável ter ciência da realidade das pessoas com os quais se deseja produzir uma ação educativa, bem como suas potencialidades e suscetibilidades de maneira completa. Dessa forma, a Educação em Saúde pode e deve ser adaptada ao contexto, aos interesses e aos conhecimentos já construídos de cada indivíduo ${ }^{11}$.

O exame revela a feminilidade nas mulheres

Nesta categoria, observou-se que algumas mulheres que realizaram o exame de PCCU e se sentiram mais femininas e apresentaram sua autoestima elevada.

O enfermeiro tem um papel fundamental nesta orientação, pois quando o profissional transmitir uma segurança para a paciente, e sanar todas suas duvidas, o grau de satisfação emocional gera resultados satisfatórios tanto para o profissional que está realizando o procedimento, quanto para a paciente que esta sendo examinada, pois elava sua autoestima fazendo se sentir mais mulher e mais segura dando mais importância aos cuidados com sua saúde.

Ao avaliar a mulher esclarecida percebeu-se que além de autoridade do corpo feminino, ela também detém um apoio para a construção da feminilidade, já que essa não seria determinada somente em forma discursiva, mas também em forma performativa mediante práticas reiteradas, como o exame. Enquanto outros métodos concebe uma possibilidade das mulheres ganharem controle sobre seus corpos ${ }^{13}$.

No ato das entrevistas observou-se que algumas mulheres se sentiam mais forte com a realização do exame e com os resultados positivos. Isso demonstra que no aspecto cultural e psicológico influencia bastante dando mais importância ao exame de PCCU. 
[...] importante de saber que não tenho nada, e que o exame de mama também mostrar se tem alguma coisa diferente, e esse se estou bem, e importante. (Diamante)

No depoimento de Diamante, tornou-se perceptivo que o exame a possibilita uma melhoria na QV de forma a trazer-lhe felicidade, segurança e harmonia, pois no seu entendimento o exame mostra que ela é uma mulher saudável para seu marido e responsável pela sua saúde, demonstra que a mulher valorizar sua saúde e buscar se prevenir devido os laços afetivos e a preocupação com sua família. No próximo depoimento, observa-se que a prevenção é o foco da Estratégia Saúde da Família, pois a mesma se preocupa com o tempo de acometimento da doença, bem como com o tratamento.

[...] a importância é de que quanto antes se descobrir maior são as chances de se tratar, $e$ quando eu faço o exame eu me sinto mais mulher, mais firme, mais forte, por se saber que não tenho nenhuma doença maligna, pra mim e por isso que e importante. (Esmeralda)

No depoimento de Esmeralda, ela mostra-se informada na questão do tratamento imediato, também se sente mais forte, mais encorajada ao realizar o exame e tem sua autoestima elevada ao saber que não tem nenhum tipo de patologia a ser tratada.

As orientações e o exame são focados na prevenção

Nesta categoria observamos que muitas mulheres dão importância ao exame de PCCU devido às campanhas preventivas divulgadas no meio de comunicação com rádio e televisão.

Nos dias de hoje à implantação da educação e saúde dentro das sociedades se torna mais fácil com o apoio dos meios de comunicação rádio e televisão que tem como função mobilizar e divulgar campanhas de incentivos de prevenção à saúde. Isso se reflete diretamente na sociedade que busca mais informação nas unidades de saúde de como se prevenir.

É imprescindível orientá-las através das práticas educativas, abordando temas incluídos à prevenção, em que as mensagens devem ser claras, objetivas e numa linguagem adaptada aos padrões culturais linguísticos das mesmas. É indispensável apresentar e revelar as possibilidades que o sistema de saúde disponibiliza para que possam usufruí-las em prol do alcance de uma melhor condição de saúde ${ }^{14-16}$.

Por meio das entrevistas, identificou-se que algumas mulheres já tinham consciência da importância do exame através dos meios de comunicação e dos agentes de saúde, e com base nesse 
ISSN 2179-6750

conhecimento que o enfermeiro deve explorar e orienta o paciente.

[...] E importante sempre se prevenir, pois esse exame fica passando o tempo todo na televisão e falando que e importante que a mulher deve se cuidar fazer o acompanhamento certinho e para que se tiver alguma coisa dentro de nós a gente pode saber o que está acontecendo e tem tempo de se cuidar melhor e com mais tempo. (Cristal)

No depoimento da paciente Cristal observou-se a importância das campanhas incentivadoras pelo governo, e são transmitidas, através dos meios de comunicação que hoje são a forma mais fácil de atingir o publico alvo e da importância da visita domiciliar realizadas pelos agentes de saúde que tem como função orientar e divulgar as campanhas entre as comunidades. É o que observamos no depoimento seguinte:

[...] Na minha visão, é importante devido ser uma doença que esta matando várias mulheres, eu percebo que a enfermeira tenta nos orientar sobre todos os riscos, e que nesses riscos, estão os cânceres, doenças sexualmente transmissíveis, HPV, e que isso tudo, ocorre por a agente não se cuida e acaba morrendo por não tratar direito. E porque eu vejo muito isso no bairro que os agentes de saúde fala que e importante se cuidar e não deixar passa o dia da consulta com enfermeira pois ela pode ajudar antes de acontecer o pior. (Quartzo)

Na entrevista de Quartzo observamos que o medo de se adquirir uma doença leva a paciente a procurar entender e buscar mais informações sobre os benefícios da prevenção, isso se reflete como um ponto positivo, pois cria-se uma rotina de horário e obrigações com sua própria saúde.

\section{Considerações Finais}

Evidenciou-se a necessidade imediata da transmissão de informações sobre os riscos da não realização do exame de PCCU e de uma boa educação em saúde, criando um vínculo entre o profissional e a cliente. Através dos resultados obtidos, nessa pesquisa, percebeu-se que as mulheres possuem um conhecimento superficial a respeito do exame, e dos diversos tipos de riscos existentes e de como minimizar ou se proteger de tais riscos.

As mulheres que serão submetidas ao exame necessitam ser orientadas sobre os riscos, evitando assim a maior exposição aos elementos nocivos, preservando e gerando uma consequente QV para todas.

Acredita-se que os resultados deste estudo possam auxiliar outras pesquisas no que se refere 175 
ISSN 2179-6750

à percepção das mulheres sobre as orientações do enfermeiro no exame de Papanicolau, fomentando possíveis pesquisas e estudos que abrangem a mesma linha de raciocínio.

\section{Referências}

1. Brasil. Ministério da Saúde (MS). Instituto Nacional de Câncer (INCA). Estimativa 2012: incidência de câncer no Brasil. Rio de Janeiro: INCA; 2011. Disponível em: http://www.inca.gov.br/rbc/n_57/v04/pdf/13_resenha_estimativa2012_incidencia_de_cancer _no_brasil.pdf

2. Nogueira ACC, Silva LB. Saúde, gênero e serviço social: contribuições sobre o câncer e saúde da mulher. Vértices [Internet]. 2009 [acesso em 17 mai 2013];11(1/3):7-17. Disponível em: http://essentiaeditora.iff.edu.br/index.php/vertices/article/view/1809$2667.20090001 / 3$

3. Casarin MR, Piccoli JCE. Educação em saúde para prevenção do câncer de colo do útero em mulheres do município de Santo Ângelo/RS. Ciênc Saúde Colet [Internet]. 2011 [acesso em 12 mar 2014];16(9):3925-32. Disponível em: http://www.scielo.br/pdf/csc/v16n9/a29v16n9.pdf

4. Lucena LT, Zãn DG, Crispim PTB, Ferrari JO. Fatores que influenciam a realização do exame preventivo do câncer cérvico-uterino em Porto Velho, Estado de Rondônia, Brasil. Rev Pan-Amaz Saúde [Internet]. 2011 [acesso em 12 mar 2014];2(2):45-50. Disponível em: http://scielo.iec.pa.gov.br/pdf/rpas/v2n2/v2n2a07.pdf

5. Melo MCSC, Vilela F, Salimena AMO, Souza IEO. O enfermeiro na prevenção do câncer do colo do útero: o cotidiano da atenção primária. Rev Bras Cancerol [Internet]. 2012 [acesso em 20 abr 2014];58(3):389-98. Disponível em: http://www1.inca.gov.br/rbc/n_58/v03/pdf/08_artigo_enfermeiro_prevencao_cancer_colo_u tero_cotidiano_atencao_primaria.pdf

6. Oliveira MM, Pinto IC. Percepção das usuárias sobre as ações de prevenção do câncer do colo do útero na estratégia saúde da família em uma distrital de saúde do município de Ribeirão Preto, São Paulo, Brasil. Rev Bras Saúde Mater Infant [Internet]. 2007 [acesso em 2 abr 2014];7(1):31-8. Disponível em: http://www.scielo.br/pdf/rbsmi/v7n1/a04v07n1.pdf

7. Fonseca RMGS, Souza KV, Andrade CJM, Amaral MA, Souza V, Caetano LC. Formação de um grupo de pesquisa em enfermagem na área da saúde da mulher e gênero. Texto Contexto - Enferm [Internet]. 2012 [acesso em 20 abr 2014];21(4):990-8. Disponível em: http://www.scielo.br/pdf/tce/v21n4/32.pdf 
ISSN 2179-6750

8. Castro RCL, Knauth DR, Harzheim E, Hauser L, Duncan BB. Avaliação da qualidade da atenção primária pelos profissionais de saúde: comparação entre diferentes tipos de serviços. Cad Saúde Pública [Internet]. 2012 [acesso em 2 abr 2014];28(9):1772-84. Disponível em: http://www.scielo.br/pdf/csp/v28n9/v28n9a15.pdf

9. Minayo MCS, Gomes SFDR. (orgs.). Pesquisa social: teoria, método e criatividade. $27^{\mathrm{a}}$ ed. Petrópolis: Vozes, 2008, p.9-29.

10. Bardin L. Análise de conteúdo. Lisboa: Edições 70, 2006.

11. Roecker S, Budó MLD, Marcon SS. Trabalho educativo do enfermeiro na estratégia saúde da família: dificuldades e perspectivas de mudanças. Rev Esc Enferm USP [Internet]. 2012;46(3):641-9. Disponível em: http://www.scielo.br/pdf/reeusp/v46n3/16.pdf

12. Pimenta DN, Leandro A, Schall VT. A estética do grotesco e a produção audiovisual para a educação em saúde: segregação ou empatia? O caso das leishmanioses no Brasil. Cad Saúde Pública [Internet]. 2007 [acesso em 15 abr 2014];23(5):1161-71. Disponível em: http://www.scielo.br/pdf/csp/v23n5/18.pdf

13. Bush J. It's just a part of being woman: cervical screening, the body and femininity. Soc Sci Med [Internet]. 2000 [acesso em 20 abr 2014];50(3):429-44. Disponível em: http://www.ncbi.nlm.nih.gov/pubmed/10626766

14. Ribeiro MGM, Santos SMR, Teixeira MTB. Itinerário terapêutico de mulheres com câncer do colo do útero: uma abordagem focada na prevenção. Rev Bras Cancerol [Internet]. 2011 [acesso em 20 abr 2014];57(4):483-91. Disponível em: http://www.inca.gov.br/rbc/n_57/v04/pdf/04_artigo_itinerario_terapeutico_de_mulheres_can cer_colo_do_utero.pdf

15. Souza GDS, Oliveira RAA, Stevanin A, Sousa MF, Almeida EC. A concepção das mulheres de Mirandópolis-São Paulo acerca do exame de Papanicolau. Rev Enferm UFSM [Internet]. 2013 [acesso em 2 abr 2015];3(3):470-9. Disponível em: http://cascavel.ufsm.br/revistas/ojs-2.2.2/index.php/reufsm/article/view/9647/pdf

16. Pinho MCV, Jodas DA, Scochi MJ. Profissionais de saúde e o programa de controle do câncer de colo uterino e mama. Rev Enferm UFSM [Internet]. 2012 [acesso em 2 abr 2015];2(2):242-51. Disponível em: http://cascavel.ufsm.br/revistas/ojs2.2.2/index.php/reufsm/article/view/4418/3748 